\title{
CALORIC MEASURE FOR ARBITRARY OPEN SETS
}

\author{
NEIL A. WATSON \\ (Received 7 February 2011; accepted 8 February 2011; first published online 4 March 2013) \\ Communicated by P. C. Fenton
}

\begin{abstract}
We give a systematic treatment of caloric measure for arbitrary open sets. The caloric measure is defined only on the essential boundary of the set. Our main result gives criteria for the resolutivity of essential boundary functions, and their integral representation in terms of caloric measure. We also characterize the caloric measure null sets in terms of the boundary singularities of nonnegative supertemperatures.
\end{abstract}

2010 Mathematics subject classification: primary 35K05; secondary 31B10, $31 \mathrm{~B} 20$.

Keywords and phrases: hypotemperature, hypertemperature, caloric measure, Dirichlet problem.

\section{Introduction}

Caloric measure is sometimes called harmonic measure for the heat equation, sometimes parabolic measure. It has been studied by many authors for particular boundaries, for example in [2, 4-14, 18-20]. However, to my knowledge it has never been given a systematic treatment for arbitrary open sets. In this paper we give such a treatment, guided by the treatment for Laplace's equation given in [1]. As usual, the different behaviour of the temporal variable and the different form of the maximum principle cause problems not encountered in the classical case.

We follow [16] in our definition of the essential boundary, and hence of the Dirichlet problem itself. This seems to be the most elementary and natural way to proceed. Assuming only that there is a caloric measure on the boundary, Suzuki [15] has proved that it must be supported by the essential boundary. In our treatment, the caloric measure is defined only on the essential boundary from the outset.

As in [16], the method of solution of the Dirichlet problem is that of PerronWiener-Brelot (PWB). In Section 3 we present a series of new and essential lemmas regarding upper and lower solutions. Caloric measure is introduced in Section 4, which is mainly concerned with the resolutivity of essential boundary functions, and their integral representation in terms of caloric measures. This culminates in our main result

(c) 2013 Australian Mathematical Publishing Association Inc. 1446-7887/2013 \$16.00 
and its two corollaries, the latter of which gives equivalent conditions for resolutivity in terms of caloric measure. Finally, in Section 5, we characterize the caloric measure null sets in terms of the boundary singularities of nonnegative supertemperatures.

\section{Preliminaries and the classification of boundary points}

We use $E$ to denote an arbitrary open subset of $\mathbb{R}^{n+1}=\left\{(x, t): x \in \mathbb{R}^{n}, t \in \mathbb{R}\right\}$. The $x$ variables may be referred to as the spatial variables, and the $t$ as the temporal variable. All of our functions are extended-real valued.

Definition 2.1. For each $(x, t) \in \mathbb{R}^{n+1}$ and $c>0$, we put

$$
\Delta(x, t ; c)=B(x, \sqrt{c}) \times] t-c, t[
$$

where $B(x, \sqrt{c})$ denotes the open ball in $\mathbb{R}^{n}$ with centre $x$ and radius $\sqrt{c}$. We call $\Delta(x, t ; c)$ the heat cylinder with centre $(x, t)$ and radius $c$. The set

$$
\partial_{n} \Delta(x, t ; c)=(B(x, \sqrt{c}) \times\{t-c\}) \cup(\partial B(x, \sqrt{c}) \times[t-c, t])
$$

is called the normal boundary of $\Delta(x, t ; c)$.

Definition 2.2. The mean value over normal boundary of the heat cylinder is defined, for any function $u$ such that the integral exists, by

$$
\mathcal{L}(u ; x, t ; c)=\int_{\partial_{n} \Delta(x, t ; c)} u d \mu_{(x, t)},
$$

where $\mu_{(x, t)}$ is the caloric measure at $(x, t)$ for $\Delta(x, t ; c)$.

Since the caloric measure is invariant under translation and parabolic dilation, the mean $\mathcal{L}(u ; x, t ; c)$ depends only on $u,(x, t)$ and $c$.

Note that, by taking $u=1$ in $[17$, Theorem 4$]$, we obtain $\mathcal{L}(1 ; x, t ; c)=1$ for all $(x, t)$ and $c$.

Given a point $p_{0} \in E$, we denote by $\Lambda\left(p_{0} ; E\right)$ the set of points $p$ for which there is a polygonal path in $E$ that joins $p_{0}$ to $p$, along which the temporal variable is strictly decreasing. By a polygonal path, we mean a path which is the union of finitely many line segments.

We also denote by $\Lambda^{*}\left(p_{0} ; E\right)$ the set of points $p$ for which there is a polygonal path in $E$ joining $p_{0}$ to $p$, along which the temporal variable is strictly increasing.

We define hypotemperatures in terms of the means $\mathcal{L}$, in line with [17].

Definition 2.3. Let $w$ be an upper finite and upper semicontinuous function on $E$. Suppose that, given any point $p \in E$, the inequality $w(p) \leq \mathcal{L}(w ; p ; c)$ holds whenever $\bar{\Delta}(p ; c) \subseteq E$. Then $w$ is called a hypotemperature on $E$. If, in addition, $w(p)>-\infty$ on a dense subset of $E$, then $w$ is called a subtemperature on $E$.

Definition 2.4. If $-w$ is a hypotemperature on $E$, we call $w$ a hypertemperature on $E$. If $-w$ is a subtemperature, we call $w$ a supertemperature. 
Lemma 2.5. If $w$ is a hypotemperature on $E$, and $w(p)>-\infty$ for some point $p \in E$, then $w$ is a subtemperature on $\Lambda(p ; E)$.

Proof. Theorem 20 of [17] holds with $\left(\delta_{3}\right)$ omitted and 'subtemperature' replaced by 'hypotemperature' throughout. It therefore follows from [17, Lemma 9] that $w$ is locally integrable on $\Lambda(p ; E)$, and hence finite on a dense subset of $\Lambda(p ; E)$.

We now classify the various types of boundary point of $E$, using the following notations for the upper and lower half-balls. Given any point $p_{0}=\left(x_{0}, t_{0}\right) \in \mathbb{R}^{n+1}$ and $r>0$, we denote by $H\left(p_{0}, r\right)$ the open lower half-ball

$$
\Lambda\left(p_{0} ; B\left(p_{0}, r\right)\right)=\left\{(x, t):\left|x-x_{0}\right|^{2}+\left(t-t_{0}\right)^{2}<r^{2}, t<t_{0}\right\},
$$

and by $H^{*}\left(p_{0}, r\right)$ the open upper half-ball

$$
\Lambda^{*}\left(p_{0} ; B\left(p_{0}, r\right)\right)=\left\{(x, t):\left|x-x_{0}\right|^{2}+\left(t-t_{0}\right)^{2}<r^{2}, t>t_{0}\right\} .
$$

Throughout this paper, the boundary of $E$ is taken relative to the one-point compactification of $\mathbb{R}^{n+1}$. Thus $\partial E$ contains the point at infinity if and only if $E$ is unbounded. Our classification is the same as that in [16], although some of the terminology and notation are different.

Definition 2.6. Let $q \in \partial E$. We call $q$ a normal boundary point if either:

(a) $q$ is the point at infinity; or

(b) $q \in \mathbb{R}^{n+1}$, and for every $r>0, H(q, r) \backslash E \neq \emptyset$.

Otherwise, we call $q$ an abnormal boundary point.

If $q$ is an abnormal boundary point of $E$, there is some $r_{0}>0$ such that $H\left(q, r_{0}\right) \subseteq E$, and we define $\Lambda(q ; E)=\Lambda\left(q ; E \cup B\left(q, r_{0}\right)\right)$. The abnormal boundary points are of the following two kinds, according to whether they can be approached from above by points in $E$.

Definition 2.7. If there is some $r_{1}<r_{0}$ such that $H^{*}\left(q, r_{1}\right) \cap E=\emptyset$, then $q$ is called a singular boundary point. In this case, $H\left(q, r_{1}\right)=B\left(q, r_{1}\right) \cap E$. On the other hand if, for every $r<r_{0}$, we have $H^{*}(q, r) \cap E \neq \emptyset$, then $q$ is called a semisingular boundary point.

The set of all normal boundary points of $E$ is denoted by $\partial_{n} E$, that of all abnormal points by $\partial_{a} E$, that of all singular points by $\partial_{s} E$, and that of all semisingular points by $\partial_{s s} E$. Thus $\partial E=\partial_{n} E \cup \partial_{a} E$ and $\partial_{a} E=\partial_{s} E \cup \partial_{s s} E$.

Definition 2.8. The essential boundary $\partial_{e} E$ is defined by

$$
\partial_{e} E=\partial_{n} E \cup \partial_{s s} E=\partial E \backslash \partial_{s} E .
$$

Lemma 2.9. Let $p_{0} \in E$, and put $\Lambda=\Lambda\left(p_{0} ; E\right)$. Then $\partial_{e} \Lambda \subseteq \partial_{e} E$ and $\partial_{s s} \Lambda \subseteq \partial_{s s} E$. If $q \in \partial_{n} \Lambda \cap \partial_{a} E$, there is an open half-ball $H\left(q, r_{1}\right)$ such that $H\left(q, r_{1}\right) \cap \Lambda=\emptyset$. 
Proof. The first two parts are given in [16, Lemma 1], and its corollary.

For the last part, since $q \in \partial_{a} E$, there is an open half-ball $H\left(q, r_{0}\right) \subseteq E$. If there was a sequence $\left\{q_{k}\right\}$ in $H\left(q, r_{0}\right) \cap \Lambda$ such that $q_{k} \rightarrow q$ as $k \rightarrow \infty$, we would have

$$
\Lambda\left(q_{k} ; H\left(q, r_{0}\right)\right) \subseteq \Lambda\left(q_{k} ; E\right) \subseteq \Lambda\left(p_{0} ; E\right)=\Lambda
$$

for all $k$, which implies that

$$
H\left(q, r_{0}\right)=\bigcup_{k=1}^{\infty} \Lambda\left(q_{k} ; H\left(q, r_{0}\right)\right) \subseteq \Lambda,
$$

contrary to the hypothesis that $q \in \partial_{n} \Lambda$. Hence there is no such sequence, and so there is $r_{1}<r_{0}$ such that $H\left(q, r_{1}\right) \cap \Lambda=\emptyset$.

\section{Upper and lower PWB solutions}

The next definition details what we mean by the Dirichlet problem for the heat equation. It is the same as that in [16], but different from that in, for example, [3].

Definition 3.1. Let $f \in C\left(\partial_{e} E\right)$. We say that a temperature $u$ on $E$ is a classical solution of the Dirichlet problem for $f$ if both

$$
\lim _{(x, t) \rightarrow(y, s)} u(x, t)=f(y, s) \quad \text { for all }(y, s) \in \partial_{n} E
$$

and

$$
\lim _{(x, t) \rightarrow(y, s+)} u(x, t)=f(y, s) \quad \text { for all }(y, s) \in \partial_{s s} E .
$$

Given this definition, our upper and lower classes for the PWB method are as given in the following definition.

Definition 3.2. Let $f$ be a function on $\partial_{e} E$. The upper class determined by $f$, denoted by $\mathfrak{U}_{f}^{E}$, consists of all lower bounded hypertemperatures on $E$ that satisfy

$$
\liminf _{(x, t) \rightarrow(y, s)} w(x, t) \geq f(y, s) \quad \text { for all }(y, s) \in \partial_{n} E,
$$

and

$$
\liminf _{(x, t) \rightarrow(y, s+)} w(x, t) \geq f(y, s) \quad \text { for all }(y, s) \in \partial_{s s} E .
$$

The lower class determined by $f$, denoted by $\mathfrak{Q}_{f}^{E}$, consists of all upper bounded hypotemperatures on $E$ that satisfy

$$
\limsup _{(x, t) \rightarrow(y, s)} w(x, t) \leq f(y, s) \quad \text { for all }(y, s) \in \partial_{n} E,
$$

and

$$
\limsup _{(x, t) \rightarrow(y, s+)} w(x, t) \leq f(y, s) \quad \text { for all }(y, s) \in \partial_{s s} E .
$$


Definition 3.3. The function $U_{f}^{E}=\inf \left\{w: w \in \mathfrak{U}_{f}^{E}\right\}$ is called the upper solution for $f$ on $E$, and $L_{f}^{E}=\sup \left\{w: w \in \mathfrak{Q}_{f}^{E}\right\}$ is called the lower solution for $f$ on $E$.

For elementary results about upper and lower solutions, the reader is referred to [16, Lemmas 24 and 26].

Let $p_{0} \in E$, and put $\Lambda=\Lambda\left(p_{0} ; E\right)$. Then $\partial_{e} \Lambda \subseteq \partial_{e} E$ by Lemma 2.9. Therefore, if $f$ is a function on $\partial_{e} E$, the classes $\mathfrak{U}_{f}^{\Lambda}$ and $\mathfrak{Q}_{f}^{\Lambda}$ are defined. Our next lemma shows that these classes are related in a convenient and natural way.

Lemma 3.4. Let $f$ be a function on $\partial_{e} E$, let $p_{0} \in E$, and put $\Lambda=\Lambda\left(p_{0} ; E\right)$. Then $\mathfrak{U}_{f}^{\Lambda}$ is precisely the class of restrictions to $\Lambda$ of the members of $\mathfrak{U}_{f}^{E}$, and $\mathfrak{Q}_{f}^{\Lambda}$ is that of the restrictions to $\Lambda$ of the members of $\mathfrak{Q}_{f}^{E}$. Hence $U_{f}^{\Lambda}$ is the restriction to $\Lambda$ of $U_{f}^{E}$, and $L_{f}^{\Lambda}$ is that of $L_{f}^{E}$.

Proof. The result for the lower classes and lower solutions is the dual of that for the upper classes and upper solutions, so we give details only for the latter.

Given any hypertemperature $w \in \mathfrak{U}_{f}^{\Lambda}$, we define a function $\bar{w}$ on $E$ by putting

$$
\bar{w}(p)= \begin{cases}w(p) & \text { if } p \in \Lambda, \\ +\infty & \text { if } p \in E \backslash \bar{\Lambda} \\ \liminf _{q \rightarrow p, q \in \Lambda} w(q) & \text { if } p \in \partial \Lambda \cap E .\end{cases}
$$

We claim that $\bar{w} \in \mathfrak{U}_{f}^{E}$. Clearly $\bar{w}$ is lower semicontinuous and lower bounded on $E$. It remains to show that, given any point $p \in E$ and any $\epsilon>0$, we can find a positive number $c<\epsilon$ such that the inequality $\bar{w}(p) \geq \mathcal{L}(\bar{w} ; p ; c)$ holds. Clearly this holds if $p \in E \backslash \partial \Lambda$, so suppose that $p \in E \cap \partial \Lambda$. Since $\partial_{e} \Lambda \subseteq \partial_{e} E$ by Lemma 2.9, $p \in \partial_{s} \Lambda$. Therefore we can find $r_{0}>0$ such that $H\left(p, 2 r_{0}\right)=B\left(p, 2 r_{0}\right) \cap \Lambda$. We now choose $c_{0}>0$ such that the closed heat cylinder $\bar{\Delta}(q ; c) \subseteq \Lambda$ whenever $q \in H\left(p, r_{0}\right)$ and $c \leq c_{0}$. Then, for any $c \leq c_{0}$,

$$
\bar{w}(p)=\liminf _{q \rightarrow p, q \in \Lambda} w(q) \geq \liminf _{q \rightarrow p, q \in \Lambda} \mathcal{L}(w ; q ; c)=\liminf _{q \rightarrow p, q \in \Lambda} \mathcal{L}(\bar{w} ; q ; c) \geq \mathcal{L}(\bar{w} ; p ; c),
$$

by Fatou's lemma and the translation invariance of the caloric measures for heat cylinders. Hence $\bar{w}$ is a hypertemperature on $E$.

We now take any point $(y, s) \in \partial_{e} E$. If $(y, s) \notin \partial \Lambda$, then

$$
\liminf _{(x, t) \rightarrow(y, s)} \bar{w}(x, t)=+\infty \geq f(y, s) .
$$

If $(y, s) \in \partial_{s} \Lambda$ then $(y, s) \in \partial_{s s} E$ and

$$
\liminf _{(x, t) \rightarrow(y, s+)} \bar{w}(x, t)=+\infty \geq f(y, s) .
$$

If $(y, s) \in \partial_{s s} \Lambda$, then $(y, s) \in \partial_{s s} E$ by Lemma 2.9, and

$$
\liminf _{(x, t) \rightarrow(y, s+)} \bar{w}(x, t)=\liminf _{(x, t) \rightarrow(y, s+)} w(x, t) \geq f(y, s) .
$$


If $(y, s) \in \partial_{n} \Lambda$, then

$$
\liminf _{(x, t) \rightarrow(y, s)} \bar{w}(x, t)=\liminf _{(x, t) \rightarrow(y, s)} w(x, t) \geq f(y, s) .
$$

Hence $\bar{w} \in \mathfrak{U}_{f}^{E}$, and so $w$ is the restriction to $\Lambda$ of a function in $\mathfrak{l}_{f}^{E}$.

We now show that, given any hypertemperature $v \in \mathfrak{U}_{f}^{E}$, its restriction to $\Lambda$ belongs to $\mathfrak{U}_{f}^{\Lambda}$. Obviously $v$ is a lower bounded hypertemperature on $\Lambda$. Let $q=(y, s) \in \partial_{e} \Lambda$. Then either $q \in \partial_{s s} \Lambda \subseteq \partial_{s s} E$, or $q \in \partial_{n} \Lambda \subseteq \partial_{e} E$, by Lemma 2.9. In the former case,

$$
\liminf _{(x, t) \rightarrow(y, s+),(x, t) \in \Lambda} v(x, t) \geq \liminf _{(x, t) \rightarrow(y, s+),(x, t) \in E} v(x, t) \geq f(y, s) .
$$

If $q \in \partial_{n} \Lambda \cap \partial_{n} E$, then

$$
\liminf _{(x, t) \rightarrow(y, s),(x, t) \in \Lambda} v(x, t) \geq \liminf _{(x, t) \rightarrow(y, s),(x, t) \in E} v(x, t) \geq f(y, s) .
$$

Finally, if $q \in \partial_{n} \Lambda \cap \partial_{s s} E$, then there is $\delta>0$ such that $H(q, \delta) \subseteq E$, but for all $r>0$ we have $H(q, r) \backslash \Lambda \neq \emptyset$. If there is a sequence $\left\{q_{k}\right\}$ of points in $H(q, \delta) \cap \Lambda$ such that $q_{k} \rightarrow q$ as $k \rightarrow \infty$, then

$$
\Lambda \supseteq \bigcup_{k=1}^{\infty} \Lambda\left(q_{k} ; H(q, \delta)\right)=H(q, \delta),
$$

a contradiction. There is therefore no such sequence, and hence there is a half-ball $H(q, \eta)$ contained in $\mathbb{R}^{n+1} \backslash \Lambda$. It follows that

$$
\liminf _{(x, t) \rightarrow(y, s),(x, t) \in \Lambda} v(x, t) \geq \liminf _{(x, t) \rightarrow(y, s+),(x, t) \in E} v(x, t) \geq f(y, s) .
$$

Hence the restriction of $v$ to $\Lambda$ belongs to $\mathfrak{U}_{f}^{\Lambda}$.

We now recall [17, Theorem 10]. Here, and subsequently, by a circular cylinder we mean a set of the form $B \times] a, b$, where $B$ is a ball in $\mathbb{R}^{n}$ and $-\infty<a<b<+\infty$. The result states that, if $w$ is a subtemperature on $E$, and $D$ is a circular cylinder such that $\bar{D} \subseteq E$, then the Poisson integral $u$ on $\bar{D} \backslash \partial_{n} D$ of the restriction of $w$ to $\partial_{n} D$ exists, and the function $\pi_{D} w$, defined on $E$ by putting

$$
\pi_{D} w= \begin{cases}u & \text { on } \bar{D} \backslash \partial_{n} D, \\ w & \text { on } E \backslash\left(\bar{D} \backslash \partial_{n} D\right),\end{cases}
$$

is a subtemperature which majorizes $w$ on $E$, and is a temperature on $\bar{D} \backslash \partial_{n} D$. This is used in the following definition, which is taken from [17, p. 210].

Definition 3.5. We call a nonempty family $\mathcal{F}$ of supertemperatures on $E$ a saturated family if it satisfies the two conditions:

(a) if $v, w \in \mathcal{F}$, then $v \wedge w \in \mathcal{F}$ (where $v \wedge w$ denotes the pointwise minimum of $v$ and $w$ );

(b) if $w \in \mathcal{F}$, and $D$ is a circular cylinder such that $\bar{D} \subseteq E$, then $\pi_{D} w \in \mathcal{F}$. 
The next lemma replaces [16, Lemma 23], whose proof I no longer find convincing. Lemma 3.6. Let $f$ be a function on $\partial_{e} E$. If there are points $p_{0}, q_{0} \in E$ such that $q_{0} \in$ $\Lambda\left(p_{0} ; E\right), U_{f}^{E}\left(p_{0}\right)<+\infty$, and $U_{f}^{E}\left(q_{0}\right)>-\infty$, then $U_{f}^{E}$ is a temperature on $\Lambda\left(q_{0} ; E\right)$.

Proof. We put $\Lambda=\Lambda\left(p_{0} ; E\right)$, and note that, by Lemma 3.4, we need to show that $U_{f}^{\Lambda}$ is a temperature on $\Lambda\left(q_{0} ; \Lambda\right)=\Lambda\left(q_{0} ; E\right)$.

Since $U_{f}^{E}\left(p_{0}\right)<+\infty$, we can find a hypertemperature $w_{0} \in \mathfrak{U}_{f}^{E}$ such that $w_{0}\left(p_{0}\right)<$ $+\infty$. By Lemma 2.5, $w_{0}$ is a supertemperature on $\Lambda$. By Lemma 3.4, the restriction of $w_{0}$ to $\Lambda$ belongs to $\mathfrak{U}_{f}^{\Lambda}$, and so we can write $U_{f}^{\Lambda}=\inf \mathcal{F}$, where $\mathcal{F}$ is the class of all supertemperatures that belong to $\mathfrak{U}_{f}^{\Lambda}$.

We show that $\mathcal{F}$ is a saturated family of supertemperatures on $\Lambda$. Let $u, v \in \mathcal{F}$. Then $u \wedge v$ is a lower bounded supertemperature on $\Lambda$. Moreover, whenever $(y, s) \in \partial_{n} \Lambda$ and $(x, t) \rightarrow(y, s)$ with $(x, t) \in \Lambda$,

$$
\lim \inf (u \wedge v)(x, t)=(\lim \inf u(x, t)) \wedge(\lim \inf v(x, t)) \geq f(y, s)
$$

and similarly whenever $(y, s) \in \partial_{s s} \Lambda$ and $(x, t) \rightarrow(y, s+)$. Hence $u \wedge v \in \mathcal{F}$. We now take any function $w \in \mathcal{F}$, and any circular cylinder $D$ such that $\bar{D} \subseteq \Lambda$. Then the function $\pi_{D} w$, defined relative to $\Lambda$, is a supertemperature on $\Lambda$, and is lower bounded by the same lower bound as $w$. Furthermore, since the compact set $\bar{D} \subseteq \Lambda$ and $\pi_{D} w=w$ on $\Lambda \backslash \bar{D}$, the boundary behaviour of $\pi_{D} w$ is the same as that of $w$. Therefore $\pi_{D} w \in \mathcal{F}$, and so $\mathcal{F}$ is a saturated family of supertemperatures on $\Lambda$.

Because $q_{0} \in \Lambda$ and $U_{f}^{\Lambda}\left(q_{0}\right)>-\infty$, it follows from [17, Theorem 11] that $U_{f}^{\Lambda}$ is a temperature on $\Lambda\left(q_{0} ; \Lambda\right)=\Lambda\left(q_{0} ; E\right)$, as required.

Corollary 3.7. Let $f$ be a function on $\partial_{e} E$. If there is a point $p_{0} \in E$ such that $L_{f}^{E}\left(p_{0}\right)$ and $U_{f}^{E}\left(p_{0}\right)$ are both finite, then $L_{f}^{E}$ and $U_{f}^{E}$ are temperatures on $\Lambda\left(p_{0} ; E\right)$.

Proof. Since $L_{f}^{E}\left(p_{0}\right)>-\infty$, we can find a hypotemperature $u \in \mathfrak{L}_{f}^{E}$ such that $u\left(p_{0}\right)>$ $-\infty$. By Lemma 2.5, $u$ is a subtemperature on $\Lambda\left(p_{0} ; E\right)$, and in particular is finite on a dense subset $F$ of $\Lambda\left(p_{0} ; E\right)$. Therefore $-\infty<u(q) \leq L_{f}^{E}(q) \leq U_{f}^{E}(q)$ for all $q \in F$. Since $U_{f}^{E}\left(p_{0}\right)<+\infty$, it follows from Lemma 3.6 that $U_{f}^{E}$ is a temperature on $\bigcup_{q \in F} \Lambda(q ; E)=\Lambda\left(p_{0} ; E\right)$.

Applying this result to $-f$, we obtain the result for $L_{f}^{E}$.

Lemma 3.8. Let $\left\{f_{j}\right\}$ be an increasing sequence of functions on $\partial_{e} E$, let $f=\lim _{j \rightarrow \infty} f_{j}$, and suppose that $U_{f_{m}}^{E}>-\infty$ on $E$ for some $m$. If $p_{0}$ is a point in $E$ such that $U_{f_{j}}^{E}\left(p_{0}\right)<+\infty$ for all $j$, then

$$
U_{f}^{E}=\lim _{j \rightarrow \infty} U_{f_{j}}^{E}
$$

on $\Lambda\left(p_{0} ; E\right)$.

Proof. The sequence $\left\{U_{f_{j}}^{E}\right\}$ is increasing on $E$, and $U_{f_{j}}^{E} \leq U_{f}^{E}$ on $E$ for all $j$. Therefore $\lim _{j \rightarrow \infty} U_{f_{j}}^{E} \leq U_{f}^{E}$ on $E$, and we may suppose that $U_{f_{j}}^{E}>-\infty$ on $E$ for all $j$. 
Suppose that $p_{0} \in E$ and $U_{f_{j}}\left(p_{0}\right)<+\infty$ for all $j$. For each $j$, Lemma 3.6 and our supposition that $U_{f_{j}}^{E}>-\infty$ on $E$ imply that $U_{f_{j}}^{E}$ is a temperature on $\Lambda(p ; E)$ for all $p \in \Lambda\left(p_{0} ; E\right)$, and thus on $\Lambda\left(p_{0} ; E\right)$ itself. We put $\Lambda=\Lambda\left(p_{0} ; E\right)$, and note that by Lemma 3.4, $U_{f_{j}}^{E}=U_{f_{j}}^{\Lambda}$ on $\Lambda$. We take any $p_{1} \in \Lambda$ and any $\epsilon>0$. For each $j$, we can find a hypertemperature $w_{j} \in \mathfrak{U}_{f_{j}}^{\Lambda}$ such that

$$
w_{j}\left(p_{1}\right)-U_{f_{j}}^{\Lambda}\left(p_{1}\right)<2^{-j} \epsilon
$$

Since each function $U_{f_{j}}^{\Lambda}$ is a temperature on $\Lambda, \lim _{j \rightarrow \infty} U_{f_{j}}^{\Lambda}$ is a hypertemperature on $\Lambda$. Moreover, since each function $w_{j}-U_{f_{j}}^{\Lambda}$ is a nonnegative hypertemperature on $\Lambda$, the same is true of $\sum_{j=1}^{\infty}\left(w_{j}-U_{f_{j}}^{\Lambda}\right)$, and hence of the function

$$
v=\lim _{j \rightarrow \infty} U_{f_{j}}^{\Lambda}+\sum_{j=1}^{\infty}\left(w_{j}-U_{f_{j}}^{\Lambda}\right) .
$$

For each $k$,

$$
v \geq U_{f_{k}}^{\Lambda}+\left(w_{k}-U_{f_{k}}^{\Lambda}\right)=w_{k},
$$

so that $v$ is lower bounded on $\Lambda$ and

$$
\liminf _{p \rightarrow q} v(p) \geq f_{k}(q)
$$

for all $q \in \partial_{e} \Lambda$, where the limits are taken in the appropriate sense according to whether $q \in \partial_{n} \Lambda$ or $q \in \partial_{s s} \Lambda$. It follows that (in the appropriate sense)

$$
\liminf _{p \rightarrow q} v(p) \geq f(q)
$$

for all $q \in \partial_{e} \Lambda$, so that $v \in \mathfrak{U}_{f}^{\Lambda}$ and hence $v \geq U_{f}^{\Lambda}$. In particular,

$$
U_{f}^{\Lambda}\left(p_{1}\right) \leq v\left(p_{1}\right) \leq \lim _{j \rightarrow \infty} U_{f_{j}}^{\Lambda}\left(p_{1}\right)+\sum_{j=1}^{\infty} 2^{-j} \epsilon=\lim _{j \rightarrow \infty} U_{f_{j}}^{\Lambda}\left(p_{1}\right)+\epsilon .
$$

This holds for all $\epsilon>0$, so that

$$
U_{f}^{\Lambda}\left(p_{1}\right) \leq \lim _{j \rightarrow \infty} U_{f_{j}}^{\Lambda}\left(p_{1}\right) \leq U_{f}^{\Lambda}\left(p_{1}\right) .
$$

Since $p_{1}$ is an arbitrary point of $\Lambda$, the result is established.

Definition 3.9. We say that a function $f$ on $\partial_{e} E$ is resolutive for $E$ if $L_{f}^{E}=U_{f}^{E}$ and is a temperature on $E$. In this case, we define $S_{f}^{E}=L_{f}^{E}=U_{f}^{E}$ to be the $P W B$ solution for $f$ on $E$.

Lemma 3.10. Let $f$ be a function on $\partial_{e} E$. If, for each point $q \in E$, we can find a point $p \in \Lambda^{*}(q ; E)$ such that $L_{f}^{E}(p)$ and $U_{f}^{E}(p)$ are equal and finite, then $f$ is resolutive for $E$. 
Proof. Because $L_{f}^{E}(p)$ and $U_{f}^{E}(p)$ are both finite, Corollary 3.7 shows that $L_{f}^{E}$ and $U_{f}^{E}$ are temperatures on the neighbourhood $\Lambda(p ; E)$ of the arbitrary point $q \in E$, and hence on the whole of $E$. Therefore the function $v=L_{f}^{E}-U_{f}^{E}$ is a nonpositive temperature on $E$. Since $v(p)=0$, it follows from the strong maximum principle that $v=0$ on $\Lambda(p ; E)$, and hence $v(q)=0$. Thus $v=0$ on $E$, and so $f$ is resolutive for $E$.

\section{The caloric measure on the essential boundary}

Given any subset $S$ of $\mathbb{R}^{n+1}$, we denote by $C(S)$ the class of all continuous, real valued functions on $S$.

The caloric measure arises in a familiar way.

THEOREM 4.1. Given any point $p \in E$, there is a unique nonnegative Borel measure $\mu_{p}^{E}$ on $\partial_{e} E$ such that the equality

$$
S_{f}^{E}(p)=\int_{\partial_{e} E} f d \mu_{p}^{E}
$$

holds for every $f \in C\left(\partial_{e} E\right)$. Moreover, $\mu_{p}^{E}\left(\partial_{e} E\right)=1$.

Proof. Any function $f \in C\left(\partial_{e} E\right)$ has a PWB solution $S_{f}^{E}$ on $E$, by [16, Theorem 32]. If $f, g \in C\left(\partial_{e} E\right)$ and $\alpha, \beta \in \mathbb{R}$, then

$$
S_{\alpha f+\beta g}^{E}=S_{\alpha f}^{E}+S_{\beta g}^{E}=\alpha S_{f}^{E}+\beta S_{g}^{E},
$$

so that the mapping $f \mapsto S_{f}^{E}(p)$ is a linear functional on $C\left(\partial_{e} E\right)$. Furthermore, if $f \geq 0$ and $w \in \mathfrak{U}_{f}^{E}$, then

$$
\limsup _{(x, t) \rightarrow(y, s)} w(x, t) \geq f(y, s) \geq 0
$$

for all $(y, s) \in \partial_{n} E$, and

$$
\limsup _{(x, t) \rightarrow(y, s+)} w(x, t) \geq f(y, s) \geq 0
$$

for all $(y, s) \in \partial_{s s} E$, so that $w \geq 0$ on $E$ by [16, Theorem 30]. Hence $U_{f}^{E} \geq 0$ on $E$, so that the linear functional is nonnegative. It now follows from the Riesz representation theorem that there is a unique nonnegative Borel measure $\mu_{p}^{E}$ on $\partial_{e} E$ such that $S_{f}^{E}(p)=\int_{\partial_{e} E} f d \mu_{p}^{E}$ for every $f \in C\left(\partial_{e} E\right)$. In particular, if $f(q)=1$ for all $q \in \partial_{e} E$, then $S_{f}^{E}=1$ on $E$, so that $1=S_{f}^{E}(p)=\int_{\partial_{e} E} d \mu_{p}^{E}=\mu_{p}^{E}\left(\partial_{e} E\right)$.

Definition 4.2. Given any point $p \in E$, the completion of the measure $\mu_{p}^{E}$ of Theorem 4.1 is called the caloric measure relative to $E$ and $p$. It will also be denoted by $\mu_{p}^{E}$. A function on $\partial_{e} E$ will be called $\mu_{p}^{E}$-measurable if it is measurable with respect to the completed measure.

Lemma 4.3. Let $p_{0} \in E$, and put $\Lambda=\Lambda\left(p_{0} ; E\right)$. Then for any point $p \in \Lambda$, the caloric measure $\mu_{p}^{E}$ is supported in $\partial_{e} \Lambda$, and $\mu_{p}^{\Lambda}$ is the restriction to $\partial_{e} \Lambda$ of $\mu_{p}^{E}$. 
Proof. Applying Theorem 4.1 on $\Lambda$,

$$
S_{f}^{\Lambda}(p)=\int_{\partial_{e} \Lambda} f d \mu_{p}^{\Lambda}
$$

for any point $p \in \Lambda$ and any function $f \in C\left(\partial_{e} \Lambda\right)$. Since $\partial_{e} \Lambda$ is a closed subset of $\partial_{e} E$, we can extend any such $f$ to a function $\bar{f} \in C\left(\partial_{e} E\right)$. Then an application of Theorem 4.1 on $E$ gives

$$
S_{\bar{f}}^{E}(p)=\int_{\partial_{e} E} \bar{f} d \mu_{p}^{E} .
$$

By Lemma 3.4, $S_{\bar{f}}^{E}(p)=S_{f}^{\Lambda}(p)$ for $p \in \Lambda$, and hence

$$
S_{f}^{\Lambda}(p)=\int_{\partial_{e} E} \bar{f} d \mu_{p}^{E} .
$$

This equality is independent of the choice of $\bar{f}$, so that $\mu_{p}^{E}\left(\partial_{e} E \backslash \partial_{e} \Lambda\right)=0$ and

$$
S_{f}^{\Lambda}(p)=\int_{\partial_{e} \Lambda} f d \mu_{p}^{E}
$$

The uniqueness of the caloric measure now gives the result.

LeMma 4.4. Let $f$ be a lower finite, lower semicontinuous function on $\partial_{e} E$. Then

$$
L_{f}^{E}(p)=U_{f}^{E}(p)=\int_{\partial_{e} E} f d \mu_{p}^{E}
$$

for all $p \in E$, and if $U_{f}^{E}<+\infty$ on $E$ then $f$ is resolutive for $E$.

Proof. We take an increasing sequence $\left\{f_{j}\right\}$ of functions in $C\left(\partial_{e} E\right)$ that converges pointwise to $f$ on $\partial_{e} E$. By [16, Theorem 32 ], each function $f_{j}$ is resolutive for $E$ so that, in particular, $S_{f_{j}}^{E}$ is finite valued on $E$ for all $j$. Therefore, by Lemma 3.8,

$$
U_{f}^{E}=\lim _{j \rightarrow \infty} S_{f_{j}}^{E}
$$

on $E$. Furthermore, $S_{f_{j}}^{E} \leq L_{f}^{E}$ on $E$ for all $j$, so it follows that $U_{f}^{E} \leq L_{f}^{E}$ on $E$. Since we always have $L_{f}^{E} \leq U_{f}^{E}$ on $E$, equality holds. By Theorem 4.1, for all $p \in E$,

$$
L_{f}^{E}(p)=U_{f}^{E}(p)=\lim _{j \rightarrow \infty} S_{f_{j}}^{E}(p)=\lim _{j \rightarrow \infty} \int_{\partial_{e} E} f_{j} d \mu_{p}^{E}=\int_{\partial_{e} E} f d \mu_{p}^{E} .
$$

Finally, since $U_{f}^{E} \geq S_{f_{1}}^{E}>-\infty$ on $E$, it follows from Lemma 3.6 that $U_{f}^{E}$ is a temperature on $E$ if it is upper finite, so that $f$ is resolutive for $E$ in this case.

LeMma 4.5. Let $p_{0} \in E$, and let $f$ be a function on $\partial_{e} E$. Given any number $A>U_{f}^{E}\left(p_{0}\right)$, we can find a lower finite, lower semicontinuous function $g$ on $\partial_{e} E$, such that $f \leq g$ on $\partial_{e} E$ and $U_{g}^{E}\left(p_{0}\right)<A$. Given any number $B<L_{f}^{E}\left(p_{0}\right)$, we can find an upper finite, upper semicontinuous function $h$ on $\partial_{e} E$, such that $h \leq f$ on $\partial_{e} E$ and $L_{h}^{E}\left(p_{0}\right)>B$. 
Proof. Since $U_{f}^{E}\left(p_{0}\right)<A$, we can find a function $w \in \mathfrak{U}_{f}^{E}$ such that $w\left(p_{0}\right)<A$. We define a function $g$ on $\partial_{e} E$ by putting

$$
g(y, s)=\liminf _{(x, t) \rightarrow(y, s)} w(x, t)
$$

for all $(y, s) \in \partial_{n} E$, and

$$
g(y, s)=\liminf _{(x, t) \rightarrow(y, s+)} w(x, t)
$$

for all $(y, s) \in \partial_{s s} E$. Then $g$ is lower bounded and lower semicontinuous on $\partial_{e} E$. Since $w \in \mathfrak{U}_{f}^{E}$, we also have $g \geq f$ on $\partial_{e} E$. Finally, we note that $w \in \mathfrak{U}_{g}^{E}$, which implies that $U_{g}^{E}\left(p_{0}\right) \leq w\left(p_{0}\right)<A$.

The second part follows by applying the first part to $-f$.

TheOREM 4.6. Let $p \in E$, and let $f$ be a function on $\partial_{e} E$.

(a) If $\int_{\partial_{e} E} f d \mu_{p}^{E}$ exists, then

$$
U_{f}^{E}(p)=L_{f}^{E}(p)=\int_{\partial_{e} E} f d \mu_{p}^{E} .
$$

(b) Conversely, if $U_{f}^{E}(p)=L_{f}^{E}(p)$ and is finite, then $f$ is $\mu_{p}^{E}$-integrable (and (4.1) holds).

Proof. (a) We prove that (4.1) holds for increasingly general classes of functions.

If $f$ is the characteristic function $\chi_{A}$ of a relatively open subset $A$ of $\partial_{e} E$, then (4.1) follows from Lemma 4.4.

We denote by $\mathcal{B}$ the $\sigma$-algebra of all Borel subsets of $\partial_{e} E$, and by $\mathcal{F}$ the class of all sets $A \in \mathcal{B}$ for which (4.1) holds when $f=\chi_{A}$. We prove that $\mathcal{F}=\mathcal{B}$. We know that $\mathcal{F}$ contains all the relatively open subsets of $\partial_{e} E$, so we can prove that $\mathcal{F}=\mathcal{B}$ by showing that $\mathcal{F}$ is a $\sigma$-algebra. Clearly $\partial_{e} E \in \mathcal{F}$. Suppose that $A \in \mathcal{F}$, so that

$$
\mu_{p}^{E}(A)=\int_{\partial_{e} E} \chi_{A} d \mu_{p}^{E}=U_{\chi_{A}}^{E}(p)=L_{\chi_{A}}^{E}(p) .
$$

We denote by $A^{c}$ the complement of $A$ in $\partial_{e} E$. Then, using Theorem 4.1,

$$
\begin{aligned}
\mu_{p}^{E}\left(A^{c}\right) & =1-\mu_{p}^{E}(A)=1-U_{\chi_{A}}^{E}(p)=L_{1}^{E}(p)+L_{-\chi_{A}}^{E}(p) \leq L_{\chi_{A}^{c}}^{E}(p) \leq U_{\chi_{A}}^{E}(p) \\
& \leq U_{1}^{E}(p)+U_{-\chi_{A}}^{E}(p)=1-L_{\chi_{A}}^{E}(p)=1-\mu_{p}^{E}(A)=\mu_{p}^{E}\left(A^{c}\right) .
\end{aligned}
$$

Therefore equality holds throughout, and hence

$$
L_{\chi_{A^{c}}}^{E}(p)=U_{\chi_{A^{c}}}^{E}(p)=\mu_{p}^{E}\left(A^{c}\right)=\int_{\partial_{e} E} \chi_{A^{c}} d \mu_{p}^{E} .
$$

Thus $A^{c} \in \mathcal{F}$. We now let $\left\{F_{j}\right\}$ be an expanding sequence of sets in $\mathcal{F}$, and put $F=\bigcup_{j=1}^{\infty} F_{j}$. Then $1=L_{1}^{E} \geq L_{\chi_{F}}^{E} \geq L_{\chi_{F_{j+1}}}^{E} \geq L_{\chi_{F_{j}}}^{E} \geq L_{0}^{E}=0$ for all $j$. Since $U_{\chi_{F_{j}}}^{E}$ is finite on $E$ for all $j$, it therefore follows from Lemma 3.8 that

$$
L_{\chi_{F}}^{E}(p) \geq \lim _{j \rightarrow \infty} L_{\chi_{F_{j}}}^{E}(p)=\lim _{j \rightarrow \infty} U_{\chi_{F_{j}}}^{E}(p)=U_{\chi_{F}}^{E}(p) \geq L_{\chi_{F}}^{E}(p) .
$$


Hence

$$
L_{\chi_{F}}^{E}(p)=U_{\chi_{F}}^{E}(p)=\lim _{j \rightarrow \infty} U_{\chi_{F_{j}}}^{E}(p)=\lim _{j \rightarrow \infty} \mu_{p}^{E}\left(F_{j}\right)=\mu_{p}^{E}(F)=\int_{\partial_{e} E} \chi_{F} d \mu_{p}^{E},
$$

so that $F \in \mathcal{F}$. It follows that $\mathcal{F}$ is a $\sigma$-algebra, and hence $\mathcal{F}=\mathcal{B}$.

Now we extend (4.1) to the characteristic functions of all $\mu_{p}^{E}$-measurable sets. Let $A$ be a $\mu_{p}^{E}$-measurable set. Then we can write $A=F \cup Y$ for some Borel set $F$ and some subset $Y$ of a Borel set $Z$ with $\mu_{p}^{E}(Z)=0$. Then $\mu_{p}^{E}(A)=\mu_{p}^{E}(F)$, and

$$
L_{\chi_{F}}^{E}(p) \leq L_{\chi_{A}}^{E}(p) \leq U_{\chi_{A}}^{E}(p) \leq U_{\chi_{F \cup Z}}^{E}(p) \leq U_{\chi_{F}}^{E}(p)+U_{\chi_{Z}}^{E}(p) .
$$

Since $Z, F \in \mathcal{B}$,

$$
U_{\chi Z}^{E}(p)=\int_{\partial_{e} E} \chi_{Z} d \mu_{p}^{E}=0,
$$

and (4.1) with $f=\chi_{F}$. Hence

$$
L_{\chi_{A}}^{E}(p)=U_{\chi_{A}}^{E}(p)=U_{\chi_{F}}^{E}(p)=\int_{\partial_{e} E} \chi_{F} d \mu_{p}^{E}=\mu_{p}^{E}(F)=\mu_{p}^{E}(A)=\int_{\partial_{e} E} \chi_{A} d \mu_{p}^{E} .
$$

Thus (4.1) holds with $f=\chi_{A}$.

Our next step is to extend (4.1) to all nonnegative, $\mu_{p}^{E}$-measurable, simple functions on $\partial_{e} E$. Suppose that $f$ can be written in the form $f=\sum_{i=1}^{k} \alpha_{i} \chi_{A_{i}}$, for some positive numbers $\alpha_{1}, \ldots, \alpha_{k}$ and $\mu_{p}^{E}$-measurable sets $A_{1}, \ldots, A_{k}$. Then (4.1) holds for each function $\chi_{A_{i}}$, and therefore

$$
\sum_{i=1}^{k} \alpha_{i} \mu_{p}^{E}\left(A_{i}\right)=\sum_{i=1}^{k} \alpha_{i} L_{\chi_{A_{i}}}^{E}(p) \leq L_{f}^{E}(p) \leq U_{f}^{E}(p) \leq \sum_{i=1}^{k} \alpha_{i} U_{\chi_{A_{i}}}^{E}(p) \leq \sum_{i=1}^{k} \alpha_{i} \mu_{p}^{E}\left(A_{i}\right) .
$$

Hence

$$
L_{f}^{E}(p)=U_{f}^{E}(p)=\sum_{i=1}^{k} \alpha_{i} \mu_{p}^{E}\left(A_{i}\right)=\sum_{i=1}^{k} \alpha_{i} \int_{\partial_{e} E} \chi_{A_{i}} d \mu_{p}^{E}=\int_{\partial_{e} E} f d \mu_{p}^{E},
$$

so that (4.1) holds for $f$.

We now consider the case where $f$ is an arbitrary nonnegative, $\mu_{p}^{E}$-measurable function on $\partial_{e} E$. We write $f$ as the limit of an increasing sequence $\left\{g_{j}\right\}$ of nonnegative, $\mu_{p}^{E}$-measurable, simple functions on $\partial_{e} E$. Since (4.1) holds for each function $g_{j}$,

$$
U_{g_{j}}^{E}(p)=\int_{\partial_{e} E} g_{j} d \mu_{p}^{E} \rightarrow \int_{\partial_{e} E} f d \mu_{p}^{E}
$$

Moreover,

$$
L_{f}^{E}(p) \geq \lim _{j \rightarrow \infty} L_{g_{j}}^{E}(p)=\lim _{j \rightarrow \infty} U_{g_{j}}^{E}(p) .
$$

Each function $U_{g_{j}}^{E}$ is bounded, and hence Lemma 3.8 can be used to show that $\lim _{j \rightarrow \infty} U_{g_{j}}^{E}(p)=U_{f}^{E}(p) \geq L_{f}^{E}(p)$. It follows that (4.1) holds in this case. 
Finally, we let $f$ be an arbitrary $\mu_{p}^{E}$-measurable function for which $\int_{\partial_{e} E} f d \mu_{p}^{E}$ exists. Then (4.1) holds for the positive and negative parts of $f$, so that

$$
\int_{\partial_{e} E} f d \mu_{p}^{E}=U_{f^{+}}^{E}(p)-L_{f^{-}}^{E}(p)=U_{f^{+}}^{E}(p)+U_{-f^{-}}^{E}(p) \geq U_{f}^{E}(p),
$$

and also

$$
\int_{\partial_{e} E} f d \mu_{p}^{E}=L_{f^{+}}^{E}(p)-U_{f^{-}}^{E}(p)=L_{f^{+}}^{E}(p)+L_{-f^{-}}^{E}(p) \leq L_{f}^{E}(p) \leq U_{f}^{E}(p) .
$$

Now (4.1) follows in the general case.

(b) Since $U_{f}^{E}(p)$ is finite, it follows from Lemma 4.5 that, given any positive integer $j$, we can find a lower finite, lower semicontinuous function $g_{j}$ on $\partial_{e} E$ such that $f \leq g_{j}$ on $\partial_{e} E$ and

$$
U_{g_{j}}^{E}(p)<U_{f}^{E}(p)+\frac{1}{j} .
$$

Furthermore, because $L_{f}^{E}(p)$ is finite, Lemma 4.5 also shows that we can find an upper finite, upper semicontinuous function $h_{j}$ on $\partial_{e} E$ such that $h_{j} \leq f$ on $\partial_{e} E$ and

$$
L_{h_{j}}^{E}(p)>L_{f}^{E}(p)-\frac{1}{j} .
$$

We put

$$
g=\inf _{j} g_{j}, \quad h=\sup _{j} h_{j}
$$

By Lemma 4.4,

$$
U_{f}^{E}(p)=\inf _{j} U_{g_{j}}^{E}(p)=\inf _{j} \int_{\partial_{e} E} g_{j} d \mu_{p}^{E} \geq \int_{\partial_{e} E} g d \mu_{p}^{E},
$$

and

$$
\begin{aligned}
L_{f}^{E}(p) & =-U_{-f}^{E}(p)=-\inf _{j} U_{-h_{j}}^{E}(p)=-\inf _{j} \int_{\partial_{e} E}\left(-h_{j}\right) d \mu_{p}^{E} \\
& =\sup _{j} \int_{\partial_{e} E} h_{j} d \mu_{p}^{E} \leq \int_{\partial_{e} E} h d \mu_{p}^{E} .
\end{aligned}
$$

Hence

$$
L_{f}^{E}(p) \leq \int_{\partial_{e} E} h d \mu_{p}^{E} \leq \int_{\partial_{e} E} g d \mu_{p}^{E} \leq U_{f}^{E}(p)=L_{f}^{E}(p) \in \mathbb{R},
$$

so that $h=g \mu_{p}^{E}$-almost everywhere on $\partial_{e} E$. Since $g$ and $h$ are Borel measurable, it follows that there is a Borel set $Z$ such that $\mu_{p}^{E}(Z)=0$ and $h=f=g$ on $\left(\partial_{e} E\right) \backslash Z$. All subsets of $Z$ are $\mu_{p}^{E}$-measurable, so that $f$ is a $\mu_{p}^{E}$-measurable function and

$$
L_{f}^{E}(p) \leq \int_{\partial_{e} E} f d \mu_{p}^{E} \leq U_{f}^{E}(p)=L_{f}^{E}(p) \in \mathbb{R} .
$$

Thus $f$ is $\mu_{p}^{E}$-integrable. 
Corollary 4.7. Let $f$ be a Borel measurable function on $\partial_{e} E$. If both $U_{f}^{E}$ and $L_{f}^{E}$ are finite on $E$, then $f$ is resolutive for $E$ and

$$
S_{f}^{E}(p)=\int_{\partial_{e} E} f d \mu_{p}^{E}
$$

for all $p \in E$.

Proof. We choose any point $p \in E$. If $f$ is Borel measurable, then $f^{+}$is $\mu_{p}^{E}$-measurable, so that Theorem 4.6(a) gives

$$
U_{f^{+}}^{E}(p)=L_{f^{+}}^{E}(p)=\int_{\partial_{e} E} f^{+} d \mu_{p}^{E}
$$

Since $U_{f}^{E}(p)<+\infty$, there is a hypertemperature $w \in \mathfrak{U}_{f}^{E}$ such that $w(p)<+\infty$, and since $w$ is lower bounded on $E$, there is a number $\alpha$ such that $w+\alpha \in \mathfrak{U}_{f^{+}}^{E}$. Therefore $U_{f^{+}}^{E}(p)<+\infty$, and obviously $U_{f^{+}}^{E}(p)>-\infty$. Since $p$ is an arbitrary point of $E$, Lemma 3.6 now shows that $U_{f^{+}}^{E}$ is a temperature on $E$, so that $f^{+}$is resolutive for $E$, and

$$
S_{f^{+}}^{E}(p)=\int_{\partial_{e} E} f^{+} d \mu_{p}^{E}
$$

This result holds if $f$ is replaced by $-f$. Therefore $f=f^{+}-f^{-}$is resolutive, and

$$
S_{f}^{E}(p)=S_{f^{+}}^{E}(p)-S_{f^{-}}^{E}(p)=\int_{\partial_{e} E} f^{+} d \mu_{p}^{E}-\int_{\partial_{e} E} f^{-} d \mu_{p}^{E}=\int_{\partial_{e} E} f d \mu_{p}^{E} .
$$

Corollary 4.8. Let $f$ be a function on $\partial_{e} E$. The following statements are equivalent:

(a) $f$ is resolutive for $E$;

(b) given any point $q \in E$, we can find a point $p \in \Lambda^{*}(q ; E)$ such that $f$ is $\mu_{p}^{E}$ integrable;

(c) $f$ is $\mu_{p}^{E}$-integrable for all $p \in E$.

If these statements hold, then

$$
S_{f}^{E}(p)=\int_{\partial_{e} E} f d \mu_{p}^{E}
$$

for all $p \in E$.

Proof. If statement (a) holds, then Theorem 4.6(b) shows that statement (c) holds also. If (c) holds, then obviously (b) holds too. Now suppose that (b) holds, and let $q \in E$. Then we can find a point $p \in \Lambda^{*}(q ; E)$ such that $f$ is $\mu_{p}^{E}$-integrable, so that

$$
L_{f}^{E}(p)=U_{f}^{E}(p)=\int_{\partial_{e} E} f d \mu_{p}^{E}
$$


by Theorem 4.6(a), and the integral is finite. It now follows from Lemma 3.10 that (a) holds, and so the equivalence of the three statements is established.

Finally, if statement (a) holds, then

$$
S_{f}^{E}(p)=\int_{\partial_{e} E} f d \mu_{p}^{E}
$$

for all $p \in E$, by Theorem 4.6.

It follows from Corollary 4.8 that, if $A$ is a subset of $\partial_{e} E$ which is $\mu_{p}^{E}$-measurable for all $p \in E$, then its characteristic function $\chi_{A}$ is resolutive and $S_{\chi_{A}}^{E}(p)=\mu_{p}^{E}(A)$ for all $p \in E$. Therefore, if $\mu_{p_{0}}^{E}(A)=0$ for some point $p_{0} \in E$, then $\mu_{p}^{E}(A)=0$ for all $p \in \Lambda\left(p_{0} ; E\right)$, by the minimum principle.

\section{Caloric measure null sets}

Caloric measure null sets are the negligible subsets of the essential boundary.

Definition 5.1. A subset $Z$ of $\partial_{e} E$ is called a caloric measure null set for $E$ if $\mu_{p}^{E}(Z)=0$ for all $p \in E$.

Any polar subset $Z$ of $\partial_{e} E$ is a caloric measure null set for $E$. For, by [16, Lemma 25] (which remains true if $E$ is unbounded), $U_{\chi_{Z}}^{E}=U_{0}^{E}=0$ on $E$, so that $\chi_{Z}$ is resolutive. Therefore Theorem 4.6(b) shows that $\chi_{Z}$ is $\mu_{p}^{E}$-integrable and

$$
0=U_{\chi Z}^{E}(p)=\int_{\partial_{e} E} \chi_{Z} d \mu_{p}^{E}=\mu_{p}^{E}(Z)
$$

for all $p \in E$.

If $Z$ is any caloric measure null set for $E$, then Theorem 4.6(a) shows that $\chi_{Z}$ is resolutive, with $S_{\chi Z}^{E}(p)=0$ for all $p \in E$.

We now give a criterion for a set to be a caloric measure null set in terms of the boundary singularities of supertemperatures.

THEOREM 5.2. Let $Z$ be a subset of $\partial_{e} E$. Then $Z$ is a caloric measure null set for $E$ if and only if the following condition is satisfied.

For each point $p_{0} \in E$, there is a nonnegative supertemperature $u$ on $\Lambda\left(p_{0} ; E\right)$ such that

$$
\lim _{(x, t) \rightarrow(y, s)} u(x, t)=+\infty
$$

for all points $(y, s) \in Z \cap \partial_{n} \Lambda\left(p_{0} ; E\right)$, and

$$
\lim _{(x, t) \rightarrow(y, s+)} u(x, t)=+\infty
$$

for all points $(y, s) \in Z \cap \partial_{s s} \Lambda\left(p_{0} ; E\right)$. 
Proof. We fix a point $p_{0} \in E$, and put $\Lambda=\Lambda\left(p_{0} ; E\right)$. If there is a function $u$ on $\Lambda$ as described in the statement, then for every $\epsilon>0$ we have $\epsilon u \in \mathfrak{U}_{\chi_{Z}}^{\Lambda}$. Therefore $U_{\chi_{Z}}^{\Lambda} \leq \epsilon u$, and if we make $\epsilon \rightarrow 0$ we obtain $U_{\chi z}^{\Lambda}=0$ on the dense set of points of $\Lambda$ where $u$ is finite. It now follows from Lemma 3.6 that $U_{\chi Z}^{\Lambda}$ is a temperature on $\Lambda$, so that $U_{\chi Z}^{\Lambda}(p)=0$ for all $p \in \Lambda$. By Lemma 3.4, $U_{\chi Z}^{\Lambda}$ is the restriction to $\Lambda$ of $U_{\chi Z}^{E}$, and so it follows that $U_{\chi_{Z}}^{E}=0$ on $\Lambda\left(p_{0} ; E\right)$ for every $p_{0} \in E$, and thus on $E$ itself. Now Theorem 4.6 shows that

$$
\mu_{p}^{E}(Z)=\int_{\partial_{e} E} \chi_{Z} d \mu_{p}^{E}=U_{\chi Z}^{E}(p)=0
$$

for all $p \in E$, so that $Z$ is a caloric measure null set.

Now we suppose, conversely, that $Z$ is a caloric measure null set for $E$. If $p_{0} \in E$, then $S_{\chi Z}^{E}\left(p_{0}\right)=0$ so that, for each $j \in \mathbb{N}$, we can find $u_{j} \in \mathfrak{U}_{\chi Z}^{E}$ such that $u_{j}\left(p_{0}\right)<2^{-j}$. We put $u=\sum_{j=1}^{\infty} u_{j}$ on $E$, and note that $u\left(p_{0}\right) \leq 1$. Then $u$ is a hypertemperature on $E$, and a supertemperature on $\Lambda=\Lambda\left(p_{0} ; E\right)$ by Lemma 2.5. If $(y, s) \in Z \cap \partial_{n} \Lambda$, then either $(y, s) \in \partial_{n} E$ or $(y, s) \in \partial_{s s} E$, by Lemma 2.9. In the former case,

$$
\liminf _{(x, t) \rightarrow(y, s),(x, t) \in \Lambda} u(x, t) \geq \sum_{j=1}^{\infty} \liminf _{(x, t) \rightarrow(y, s),(x, t) \in E} u_{j}(x, t)=+\infty,
$$

so that (5.1) holds. In the latter case, we put $q=(y, s)$ and note that there is an open half-ball $H\left(q, r_{1}\right)$ such that $H\left(q, r_{1}\right) \cap \Lambda=\emptyset$, by Lemma 2.9. Therefore, if $(x, t) \rightarrow(y, s)$ with $(x, t) \in \Lambda$, then $(x, t) \rightarrow(y, s+)$. Hence

$$
\liminf _{(x, t) \rightarrow(y, s),(x, t) \in \Lambda} u(x, t) \geq \sum_{j=1}^{\infty} \liminf _{(x, t) \rightarrow(y, s+),(x, t) \in E} u_{j}(x, t)=+\infty,
$$

so that (5.1) again holds. Finally, if $(y, s) \in Z \cap \partial_{s s} \Lambda$, then $(y, s) \in Z \cap \partial_{s s} E$ by Lemma 2.9, and (5.2) follows by a similar estimation.

\section{References}

[1] D. H. Armitage and S. J. Gardiner, Classical Potential Theory (Springer, London, 2001).

[2] T. Bousch and Y. Heurteaux, 'Caloric measure on domains bounded by Weierstrass-type graphs', Ann. Acad. Sci. Fenn. Math. 25 (2000), 501-522.

[3] J. L. Doob, Classical Potential Theory and its Probabilistic Counterpart, Grundlehren der mathematischen Wissenschaften, 262 (Springer, New York, 1984).

[4] E. Fabes and S. Salsa, 'Estimates of caloric measure and the initial-Dirichlet problem for the heat equation in Lipschitz cylinders', Trans. Amer. Math. Soc. 279 (1983), 635-650.

[5] E. B. Fabes, N. Garofalo and S. Salsa, 'Comparison theorems for temperatures in noncylindrical domains', Atti Accad. Naz. Lincei. Rend. Cl. Sci. Fis. Mat. Natur. 77 (1984), 1-12.

[6] Y. Heurteaux, 'Mesure harmonique et l'equation de la chaleur', Ark. Mat. 34 (1996), 119-139.

[7] S. Hofmann, J. L. Lewis and K. Nyström, 'Caloric measure in parabolic flat domains', Duke Math. J. 122 (2004), 281-346.

[8] R. Kaufman and J.-M. Wu, 'Singularity of parabolic measures', Compositio Math. 40 (1980), 243-250. 
[9] R. Kaufman and J.-M. Wu, 'Parabolic potential theory', J. Differential Equations 43 (1982), 204-234.

[10] R. Kaufman and J.-M. Wu, 'Parabolic measure on domains of class Lip $\frac{1}{2}$ ', Compositio Math. 66 (1988), 201-207.

[11] R. Kaufman and J.-M. Wu, 'Dirichlet problem of heat equation for $C^{2}$ domains', J. Differential Equations 80 (1989), 14-31.

[12] J. T. Kemper, 'Temperatures in several variables: Kernel functions, representations, and parabolic boundary values', Trans. Amer. Math. Soc. 167 (1972), 243-262.

[13] J. L. Lewis and J. Silver, 'Parabolic measure and the Dirichlet problem for the heat equation in two dimensions', Indiana Univ. Math. J. 37 (1988), 801-839.

[14] K. Nyström, 'The Dirichlet problem for second order parabolic operators', Indiana Univ. Math. J. 46 (1997), 183-245.

[15] N. Suzuki, 'On the essential boundary and supports of harmonic measures for the heat equation', Proc. Japan Acad. Ser. A Math. Sci. 56 (1980), 381-385.

[16] N. A. Watson, 'Green functions, potentials, and the Dirichlet problem for the heat equation', Proc. London Math. Soc. 33 (1976), 251-298.

[17] N. A. Watson, 'A unifying definition of a subtemperature', New Zealand J. Math. 38 (2008), 197-223.

[18] J.-M. G. Wu, 'On parabolic measures and subparabolic functions', Trans. Amer. Math. Soc. 251 (1979), 171-185; 259 (1980), 636 (erratum).

[19] J.-M. Wu, 'On heat capacity and parabolic measure', Math. Proc. Cambridge Phil. Soc. 102 (1987), 163-172.

[20] J.-M. Wu, 'An example on null sets of parabolic measures', Proc. Amer. Math. Soc. 107 (1989), 949-961.

\footnotetext{
NEIL A. WATSON, Department of Mathematics and Statistics, University of Canterbury, Private Bag, Christchurch, New Zealand e-mail: n.watson@math.canterbury.ac.nz
} 